\title{
Fabrication Du Savon Kabakrou Et Ses Conséquences Sur Les Acteurs Principaux De Gbintou Dans La Commune De Bouaké (Côte d'Ivoire)
}

\author{
Yedagne De André-Nestor \\ Université Alassane Ouattara de Bouaké (Côte d’Ivoire)
}

doi: 10.19044/esj.2016.v12n32p441 URL:http://dx.doi.org/10.19044/esj.2016.v12n32p441

\begin{abstract}
For the inhabitants of Gbintou (a district of the town of Bouaké), manufacture traditional soap, kabakrou, is the main economic activity of survival. In fact, this activity is a source of wealth, but a risky source of wealth. Indeed, the actors face many difficulties in the manufacturing process (raw material supply, health difficulties) the point of wanting to simply abandon the trade. The implementation of the socio-anthropological functionalist method and the study of this activity helped to understand that apart from raw material acquisition difficulties and illnesses it generates (contact urticaria, infections broncho lung), the company suffers from a lack of garish consequent functional organization and sanitary hygiene. Thus, it is desirable to set up an association or cooperative well equipped to train and organize stakeholders and better manage the entire manufacturing process
\end{abstract}

Keywords: Informal sector, traditional soap, unemployment, social mobility, healnes

\section{Résumé}

Pour les habitants de Gbintou (un quartier de la commune de Bouaké), la fabrication du savon traditionnel, kabakrou, est la principale activité économique de survie. A vrai dire, cette activité est une véritable source de richesse, mais une source de richesse risquée. En effet, les acteurs rencontrent de nombreuses difficultés dans le processus de fabrication (approvisionnement en matière première, des difficultés sanitaires) au point de vouloir abandonner purement et simplement le métier. L'application de la méthode socio-anthropologique et fonctionnaliste de l'étude à cette activité a permis de comprendre qu'en dehors des difficultés d'acquisition de matière première et des maladies qu'elle génère (urticaire de contact, des infections broncho-pulmonaires), l'entreprise souffre d'un manque criard 
d’organisation fonctionnelle conséquente et d’hygiène sanitaire. Ainsi, il est souhaitable de mettre en place une association ou une coopérative bien outillée pour former et organiser les acteurs et mieux gérer l’ensemble du processus de fabrication

Mots clés:Secteur informel, savon traditionnel, chômage, mobilité sociale, maladie, protection d'environnement

\section{Introduction}

Gbintou, dans la commune de Bouaké, est un quartier de population pauvre. Néanmoins ces habitants ne croisent pas les bras et s'adonnent presque tous à la fabrication du savon traditionnel, le kabakrou, pour leur survie économique depuis de nombreuses années. Cette activité qui est bien une richesse attrayante pour les habitants de Gbintou requiert du matériel de fabrication venant gratuitement de l'usine Olheol singulièrement de résidus d'huile de grains de coton par le canal des conduites d'eaux usées de l'usine. Mais les mesures de protection de l'environnement prisent par Olheol, contraignent les acteurs du kabakrou à s'approvisionner à coût d'argent auprès de cette structure et de plus en plus ailleurs, essentiellement d'huile de palme des régions sud et ouest de la Côte d'Ivoire. L'approvisionnement de cette matière première et bien d'autres produits devient de nos jours un "'casse-tête chinois"' pour tous ces acteurs. De surcroit, la manipulation des produits d'origine diverses provoque des problèmes sanitaires (urticaire de contact, infections broncho-pulmonaires). Aussi la mévente du kabakrou en cette période de l'année porte au nu les difficultés économiques des acteurs.

Dans la littérature, des sciences humaines, relative à la sociologie économique et la sociologie de la santé du secteur informel, singulièrement de la fabrication du savon traditionnel kabakrou, (MALIKWISHA, Meni., 2000; SHOMBA, Lomani., 2003 ; CUONZO, Teresa Maria., 2003 ; AKMEL Meless Siméon., 2012). Les chercheurs ont souvent mis l'accent sur la promotion, sa réforme, sa formalisation et sur la santé de ces acteurs. Mais, les études sur les difficultés que rencontrent les acteurs de la fabrication du savon kabakrou à Gbintou, sont peu ou prou mobilisées; ainsi naît notre intérêt pour cette étude.

Dès lors, nous nous demandons, pourquoi aujourd'hui les acteurs de la production du savon kabakrou sont-ils gagnés par le découragement au point d'abandonner le métier? Pourquoi les fabricants de kabakrou n'arrivent-ils pas à contourner les difficultés d'acquisition de matières premières par des stratégies renouvelées ? Quels sont les risques sanitaires qui affectent ces acteurs et qui les exaspèrent plus en plus ? Enfin, quels sont les facteurs explicatifs de la mévente du savon kabakrou à Bouaké? Bref, l'objectif de cette étude est de tenter d'analyser et de comprendre les 
facteurs qui découragent les fabricants de kabakrou de Gbintou et qui risquent l'arrêt définitif de leur activité de survie.

La thèse soutenue est la suivante : la fabrication du savon traditionnel kabakrou qui permettait aux populations de Gbintou de satisfaire leurs besoins sociaux, économiques et culturels, suscite davantage aujourd'hui, elle engendre des contraintes qui non seulement affectent négativement la production, mais engendrent le découragement chez les petits producteurs de Gbintou.

La méthode fonctionnaliste et la méthode socio-anthropologique, au sens où l'entend Olivier de $\operatorname{Sardan}^{36}$ y sont mobilisées pour donner l'éclairage, car celles-ci permettent à notre avis de maîtriser au mieux l'hétérogénéité des facteurs qui s’y interfèrent et de comprendre les difficultés que rencontrent les acteurs de la production de kabakrou. L'entretien semi-directif, premier outil de collecte des données est administré aux acteurs ayant une expérience dans la fabrication du savon. Le focus group, deuxième outil mobilisé en complément à l'entretien est essentiellement adressé aux enquêtés, pour recueillir leurs discours sur l'organisation, les facteurs qui frisent le découragement et l'arrêt définitif de la fabrication du savon kabakrou. Les enquêtés sont retenus selon les critères d'ancienneté dans le travail et selon leur disponibilité.

Ainsi pour y parvenir, nous allons dans un premier mouvement décrire les origines, la fabrication du kabakrou et mettre en exergue sa valeur socio- économique à Gbintou. Dans un deuxième mouvement, nous allons tenter d'analyser et d'expliquer les difficultés liées à la fabrication du savon kabakrou et leurs conséquences sur la survie des acteurs.

\section{Origines et fabrication du kabakrou à Gbintou Origines}

Notre site d'enquête est Gbintou, sous-quartier précaire, situé sur une pente au centre de la commune de $K o \hat{k} \hat{o}^{37}$. Il regorge différents groupes ethniques qui y exercent diverses activités (commerce, maraîcher, savon traditionnel). Le choix de la localité est lié à l’intense activité du kabakrou et sa prolifération rapide.

Les origines du savon remontent à plus de 4000 ans avant Jésus Christ. Les pâtes des savons ont des aspects bien différents de ceux des savons d'aujourd'hui et rempli une fonction médicinale. Des graisses animales ont remplacé les graisses végétales, principalement par les égyptiens. Les romains l’ont utilisé pour leurs soins quotidiens (corps,

\footnotetext{
${ }^{36}$ Olivier de Sardan., 1995a : Anthropologie et développement, Essai en Anthropologie de changement social Kartala, Paris ;

37 Selon l'INS (2013), elle compte 50.278 habitants et s'étend sur une superficie de $1475241 \mathrm{~m}^{2}$
} 
cheveux $)^{38}$. La littérature montre qu’au Ghana, avant l'arrivée des Portugais en 1482, les Fanti ${ }^{39}$ ont préparé du savon à partir de l'huile de palme brute et de la potasse, extraite des cendres de bois. L'histoire du savon kabakrou au quartier Gbintou a débuté avec l'implantation de l'usine Trituraf en 1973, devenue Olheol depuis le 18 septembre 2009. Son activité principale est la trituration de la graine de coton pour la production d'huile raffinée alimentaire d'une excellente qualité, et anti-cholestérol, vendue sous la marque Oléor, puis Eclador. L'usine traite également le soja, la graine de palmiste, le tournesol, le beurre de karité et le beurre de cacao. Elle rejette hors de ses locaux de la matière grasse à travers les conduites d'eaux usées. Cette matière, qui n'est d'aucune utilité pour cette firme constitue l'huile de base pour la fabrication du kabakrou. Ainsi, depuis 1981, l'activité est devenue une tradition qui se transmet de mère en fille, de père au fils, dans ledit quartier.

\section{Fabrication du Kabakou à Gbintou}

Á la question 'comment fabrique-t-on le kabakrou”'?, les enquêtés ont répondu :

On recueille les résidus des produits, en provenance de l'usine. Peu importe les types d'huiles utilisées. L'essentiel, c'est fabriquer le savon ; il y a aussi différents matériels employés dans la cuisson ${ }^{40}$.

Les éléments tels: la matière grasse (une variété d'huiles coton, olive, arachide, coco, palmiste, beurre de karité), la soude caustique et des ustensiles appropriés dans la production, servent à la fabrication du savon. Les acteurs de Gbintou utilisent celle, déversée par les tuyaux d'évacuation de l'usine Olheol $^{41}$, dans les eaux traversant la partie sud du quartier. En réalité, ce sont les résidus issus de la transformation du noyau de la graine de coton en huile comestible.

Première étape du processus de fabrication, la préparation consiste à 'brûler l'huile، ${ }^{42}$. La cuisson dure environ 7 h de temps, pour obtenir une substance adaptée à la fabrication du savon, après, suit la lessive de soude (mélange d'eau et de soude caustique). A ce niveau, il est impératif de toujours verser lentement la soude dans l'eau, tout en remuant la solution, jusqu'à la fonte totale de ce produit, que l'on fait reposer toute la nuit. En vue d'obtenir un produit de qualité, le respect scrupuleux des doses, à raison

\footnotetext{
${ }^{38}$ www.savonnature.com/origine-savon.php, consulté le 14/5/2015 à 10h15 mn.

${ }^{39}$ Fanti, peuple habitant le territoire de l'ancien royaume de Fanti (Ghana). Du point de vue linguistique, ils sont apparentés aux Ashanti.

${ }^{40}$ Homme 45 ans, célibataire, analphabète ; femme 38 ans, célibataire, analphabète.

${ }^{41}$ Ex-Trituraf, usine située à proximité de la société Sitab.

${ }^{42}$ Faire cuire l'huile jusqu'à obtention d'une couleur noirâtre, adaptée à la fabrication du savon. La photo ci-dessus présente la première étape de la préparation à Gbintou.
} 
de trois grandes boites de tomates pleines d'eau pour un kilogramme de soude caustique est exigé. Le lendemain, l'on procède au mélange de l'huile avec la lessive de soude, en les remuant lentement, et en observant la même quantité pour chacune d'elles, ce qui facilite la saponification.

Deuxième étape, voire la production de chaleur dans les minutes qui suivent le mélange. Mixtionner la solution aide à accélérer le processus, et permet à la soude de trouver les graisses nécessaires à la saponification. Le mélange dure 5 à 15 minutes. Pour solidifier la substance, on y ajoute parfois de la farine (blé, manioc), ce qui ouvre la voie à la trace. Elle apparait, lorsque le mélange est homogène, de couleur jaunâtre, pâteux, épais. Au niveau du modelage.

Troisième étape, les acteurs, gans plastiques à la main, façonnent le produit, en donnant au savon une forme ronde semblable à celle d'une noix de coco. Au fur et à mesure, les savons sont disposés sur un sac ou déposés dans une bassine pour être séchés.

\section{Quatrième étape, ils sont vendus.}

Les photographies suivantes montrent certaines étapes du travail de kabakrou :

\section{Photo1 : La préparation de l'huile à Gbintou}

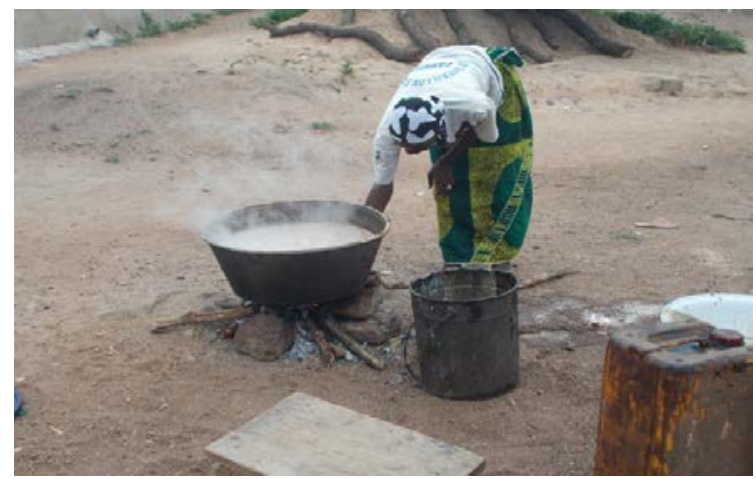

Source : Données d'enquêtes, Allah, juillet 2014. Photo 2: Le modelage de la pât

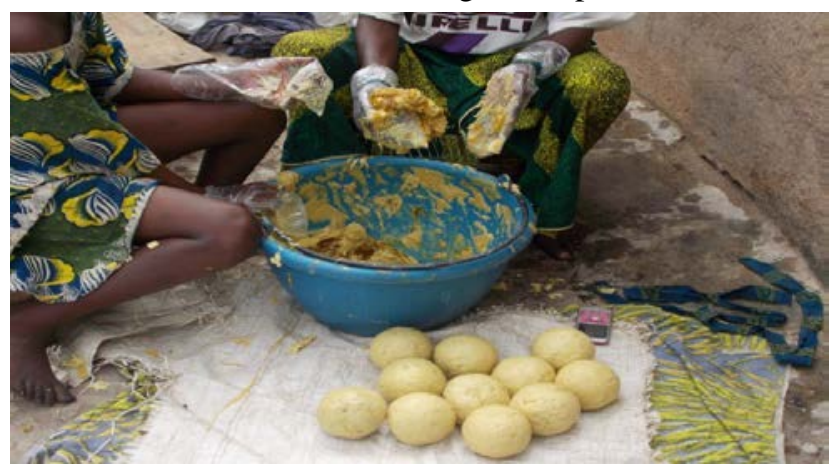

Source : Données d'enquêtes, Allah, juillet 2014. 
Sur le terrain, nous avons observé des hommes et des femmes engagés dans la production du savon traditionnel. Alors, pourquoi s’adonnent-ils à cette activité?

\section{La valeur socio-économique et la ruée vers la fabrication du savon kabakrou à Gbintou}

Pour saisir les logiques des acteurs liées à la ruée vers la fabrication du kabakrou, nous avons interrogé les producteurs. Ainsi, à la question, 'quels sont les facteurs qui motivent la fabrication du kabakrou, ils ont répondu :

La fabrication du kabakrou est devenue aujourd'hui notre source de revenus, parce qu'il se vend bien. Il peut seul supporter les dépenses; il nous permet de faire face aux charges sociales, aux cotisations de toutes sortes et à la célébration des mariages. Son rendement est quotidien et mensue $^{43}$. Les revenus de cette activité permettent d'assurer une retraite confortable et dorée, de célébrer, les mariages et le culte des mort d'enrichir le legs ancestral. Le kabakrou procure des revenus mensuels (environ $150.000 \mathrm{~F}$ par mois). Les producteurs sont payés comme des fonctionnaires ${ }^{44}$.

De ces propos découlent deux remarques, dont la première rime avec la valeur économique du travail. Le tableau ci-dessous atteste:

Graphique $n^{\circ} 1$ : Productions mensuelles de savon

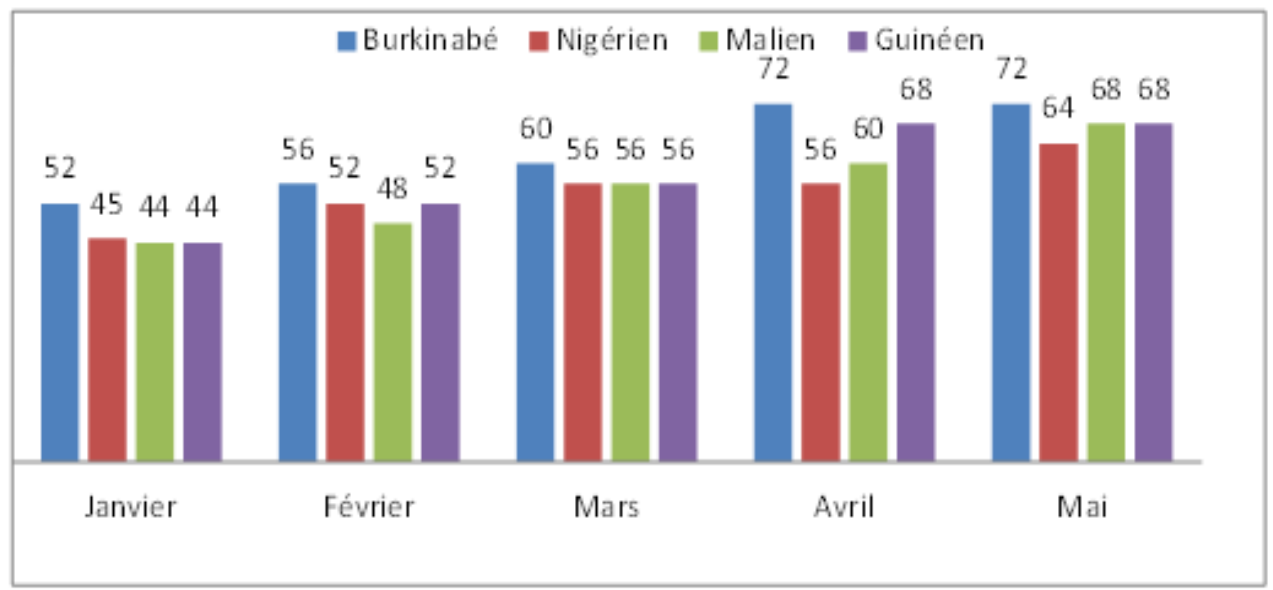

Source : Données d'enquête, Gbintou, 2014

Les données du terrain montrent une progression sensible de la production du savon traditionnel. Ses emballages sont passés de 185 sacs de $100 \mathrm{~kg}$ en janvier, à 272 paquets en mai. Ainsi chaque jour, ce sont des sacs

\footnotetext{
${ }^{43}$ Homme de nationalité malienne, 50 ans.

${ }^{44}$ Femme de nationalité burkinabé, 43 ans.
} 
de savons qui sont vendus aux grossistes de Bouaké sur le marché local (Djebonoua, Brobo, Botro, Sakassou). Mais la trajectoire du produit ne se limite pas à cette région. Elle a atteint les pays voisins, d'où sont originaires certains acteurs de la production. Deux fois la semaine, le savon est convoyé vers le Mali, le Burkina, le Niger et la Guinée. Cette hausse est justifiée par l'intensité du travail et l'implication effective des hommes et femmes dans l'activité. Cette croissance de la production a permis aux acteurs d'obtenir des revenus substantiels, ce qui est indiqué dans les tableaux suivants :

Graphique n²: Revenus mensuels à Gbintou
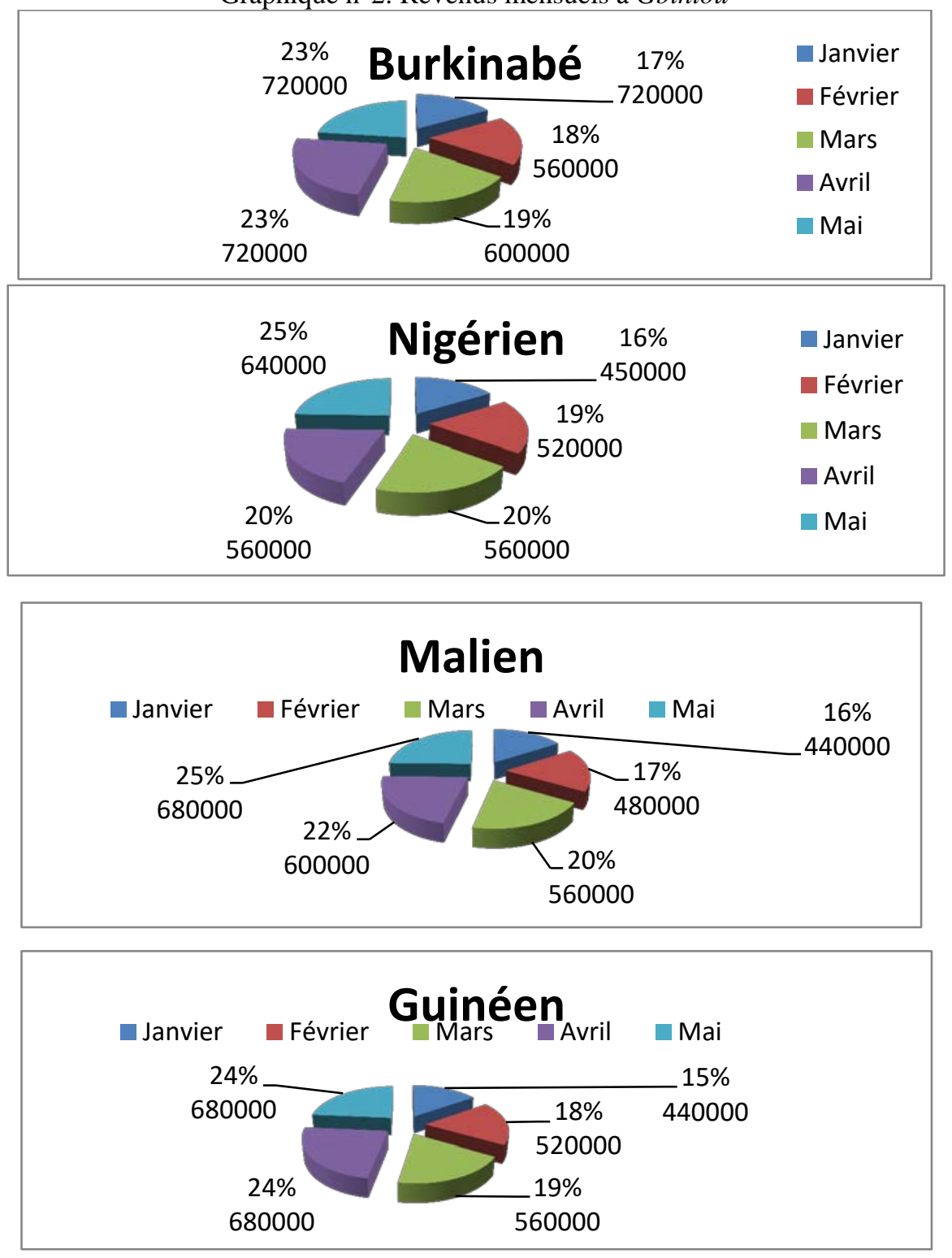

Source : Données d'enquête, 2014. 
Ces données indiquent la valeur économique qui se dégage de la fabrication du kabakrou. Ainsi, de 1.850.000 francs en janvier, ils passent à 2.720.000FCFA en mai, soit une progression de 870.000FCFA. L’avantage financier que procure cette activité est un facteur justificatif de la ruée vers 'sa fabrication. Les résultats issus des tableaux et les discours qui les accompagnent, indiquent que la fabrication de ce savon traditionnel a amélioré l'économie des populations de Gbintou. Elle est un facteur de contribution à leur développement. L’autonomie croissante des personnes qui accompagne le progrès de cette fabrication fait que nous assistons avec elle, à la naissance d'un nouveau type d'organisation sociale qui fait particulièrement appel aux relations d’intérêts. Ce dynamisme économique ou changement social est la conséquence directe de l'épanouissement des individus et de l’amélioration de leur habitat.

La deuxième remarque concerne l'importance sociale et culturelle de la commercialisation du kabakrou. L’analyse des données révèle que les revenus issus de cette activité interviennent dans les charges sociales. Ils participent à la scolarisation des enfants. En effet, face au manque criard d'emploi, et son corollaire la paupérisation, les populations se sont tournés vers la fabrication du kabakrou, en vue d'assurer les frais d'écolage, les fournitures, l'habillement, l'hébergement, la nourriture de leurs enfants. Ils s'adonnent à cette activité, parce qu'elle leur rapporte des revenus mensuels qui leur permettent de faire face aux besoins sus-énumérés.

Aussi, une expansion rapide de logements modernes est perceptible dans ce milieu. Fascinés par le modernisme, (maisons luxueuses), les acteurs du savon kabakrou s'investissent dans la construction des maisons modernes, l'achat d'appareils électroménagers, de vêtements et parures de qualité. Pour eux, la valeur de l'homme se mesure par l'acquisition ou l'accumulation de biens matériels. Des projets immobiliers ont vu le jour. Débutés en 2012, ils ont permis aux fabricants de devenir pour certains propriétaires de villas.

Il existe également une valeur culturelle liée à l’activité du kabakrou, car les revenus interviennent dans l'achat de pagnes (basins), parures, moutons, volailles destinés à la célébration des institutions sociales et religieuses comme le mariage coutumier, les baptêmes et les rites funéraires. Celles-ci participent à la solidarité, au renforcement des liens sociaux, voire la cohésion sociale. C'est une occasion pour elles de contribuer à l'enrichissement du patrimoine vestimentaire des lignages ou familles et de participer implicitement à la perpétuation de ces institutions. La célébration du mariage permet d'acquérir de nouveaux statuts, ce qui fait du récipiendaire, un homme respecté de tous. Il acquiert honneur et une position sociale ascendante, 
Somme toute, les acteurs reconnaissent que la fabrication du kabakrou génère des revenus substantiels. L’observation de certains projets immobiliers réalisés par ces fabricants justifie cette valeur économique. Les gains mensuels sont estimés à $38500 \mathrm{~F}$ (Janvier), $43000 \mathrm{~F}$ (Février), $47500 \mathrm{~F}$ (Mars), 53000 F (Avril), 56000 F (Mai), environ 238000 F par producteur. Aujourd'hui, le Kabakrou se vend bien sur les marchés intérieurs et extérieurs, à cause de son prix (100 F) et son endurance. Contrairement aux savons fabriqués dans les usines, il résiste mieux à l'eau. La majorité de la clientèle provient des ménages à revenus modestes. En vue de minimiser les dépenses, les femmes l'utilisent à plusieurs fins (vaisselle, lessive...). L'essentiel pour les populations, c'est comment assurer et assumer leur autonomie financière. De ce fait, elles luttent implicitement contre le chômage et la pauvreté, considérés comme un vécu quotidien chez les Ivoiriens, plus particulièrement chez les habitants de Gbintou. Doumbia affirme:

Les politiques et programmes d'emploi entrepris jusqu'ici en Côte d'Ivoire n'ont pas freiné la tendance, ni fait reculer l'ampleur de l'exclusion du monde du travail dont les jeunes, hommes et femmes font l'objet. L'absence d'emploi les exclue également de la protection sociale ${ }^{45}$.

Ce constat, nous permet d'affirmer que les fabricants du kabakrou sont financièrement aisés. C'est la justification de l'assertion qui indique que : le kabakrou est une spéculation très rentable en ce milieu. La ruée des populations vers ce savon s'explique par le fait que le kabakrou procure des moyens financiers incitatifs aux acteurs. Cette activité a transformé des pauvres en hommes riches. Elle leur a permis de quitter leur statut de "cancéreux économiques "'. La fabrication du kabakrou leur permet de se prendre en charge, de répondre aux besoins familiaux et aux sollicitations sociales, de scolariser les enfants et de participer au développement de leurs pays. Les revenus du kabakrou sont aussi transférés vers les pays d’origine (Burkina Faso, Guinée, Niger Mali). Venus faire fortune en Côte d'Ivoire, ces producteurs sont essentiellement des calculateurs, car les sommes d’argent amassées sont destinées à subvenir aux besoins des proches parents résidant dans les pays d'appartenance, voire améliorer leurs conditions de vie. Ces expatriés constituent un pont investissement, car l'objectif essentiel est de sortir leurs familles de la misère et participer au développement de leurs pays, peu importe les risques auxquels ils sont exposés. C'est donc à raison qu'affirme MARX :

${ }^{45}$ DOUMBIA, Vakaramoko : L'emploi des jeunes en Côte d'Ivoire, Abidjan, Université de Cocody, UFR-SEG/CIRES, 2008. 
Ce n'est pas la conscience des gens qui détermine leur être, c'est au contraire l'être social qui détermine leur conscience ${ }^{46}$. En clair, ce n'est pas la superstructure (idéologie, instruction, formation) qui préoccupe les producteurs, c’est comment exister ou vivre. Cette valeur économique du savon traditionnel, (secteur informel) semble être partagée par des chercheurs. TSHIMANKINDA atteste:

En République Démocratique du Congo, l'importance de ce secteur, surtout sous la forme du petit commerce n'est plus à démontrer. En effet, du politicien à l'homme de la rue, de l'intellectuel à l'analphabète, de l'Etat à l'individu, du citadin au paysan, tout le monde est soit opérateur, soit bénéficiaire des biens, de services fournis par ce secteur. L'informel agit aussi à la fois comme support de sécurité et amortisseur des chocs sociaux. ${ }^{47}$

Elle exprime aussi une situation sociale caractérisée par l'influence des règles sur les comportements des individus, ou un renforcement des valeurs collectives dues à l'affiliation, pour en subir 'une qualification sociale', De ce fait, la fabrication du kabakrou chez les populations de Gbintou est la courroie de transmission de la trame de celles-ci, car elle leur permet de 'disposer d'un instrument de socialisation et de principe de valeurs'. Ainsi, hommes et femmes, vieux et jeunes la considèrent comme le moteur de leur mobilité ou leur ascension sociale ${ }^{48}$. L'analyse indique ici que le kabakrou les intègre dans leur société. Il favorise le lien de transmission et l'acquisition d'une richesse sociale.

Mais cette dynamique économique, sociale et culturelle du kabakrou est très tôt entravée par des situations d'inconfort.

\section{La fabrication du kabakrou source de difficultés sociales, économiques, et sanitaires pour les populations de Gbintou}

Les prix de vente du kabakrou ont chuté de manière drastique, le gain mensuel a fortement diminué. Ce qui hypothèque leur avenir. Ainsi, l'espoir nourri par ces petits producteurs de kabakrou s’étiole. D’autres fléaux comme le problème d'approvisionnement en matière première, la mévente et les contraintes sanitaires sont permanents.

Bref à l'heure actuelle, bien des producteurs de kabakrou ne peuvent plus subvenir aux besoins de leurs familles, ni répondre aux sollicitations et cotisations pour le développement et l'épanouissement de celles-ci, d’où leur désillusion.

\footnotetext{
${ }^{46}$ MARX, Karl : Contribution à la critique de l'économie politique, Paris, Editions Sociales, 1972.

${ }^{47}$ TSHIMANKINDA, Musenga Christian : La fiscalisation de l'économie informelle comme facteur du développement économique de la République Démocratique du Congo; état des lieux et perspectives, UPC, Congo, Droit Economique et social, 2008.

${ }^{48}$ SOROKIN, Pitirim, op.cit.
} 


\section{Contraintes sociales et économiques}

Le développement des cours du savon traditionnel kabakrou fut l'élément déclencheur et catalyseur qui fit de Gbintou un lieu quasi producteur de ce savon, engendrant des profits et des dividendes qui poussaient les populations à s'aventurer dans sa production. De milliers de travailleurs de diverses origines répondirent à l'appel de la fabrication du kabakrou et se lancèrent dans l'aventure pour le commerce du précieux savon. Ainsi Gbintou connut la sensation de richesse et de prospérité. L'argent se remit à circuler, renforçant l'économie des populations. Les fabricants reçurent le surnom de 'soldats du kabakrou'. Cependant la difficulté d'approvisionnement en matières premières aujourd'hui et la mévente progressivement installée par l'introduction d'un savon liquide traditionnellement préparé, engendrent le désenchantement des populations de Gbintou.

L'une des préoccupations majeures des acteurs de kabakrou, est le problème d'approvisionnement de la matière première. En effet, La nécessité de protéger l'environnement contraint les autorités de l'usine Olheol à reconsidérer la gestion des résidus d'huiles, matières premières dans la production du savon kabakrou. Ainsi, les résidus d'huiles ne sont plus déversés dans les caniveaux d'évacuation, mais conservés dans des barils et vendus aux producteurs du savon traditionnel à 5000 ou 10.000 francs CFA l'unité ${ }^{49}$ selon les périodes. Cette mesure de protection de l'environnement met en mal l'entreprise de la production du savon kabakrou. Ces derniers la jugent contraignante financièrement, préjudiciable et presque suicidaire. Après des années de gratuités, période faste, ces fabricants doivent maintenant récupérer les résidus d'huile à prix d'argent, suite aux mesures de protection de l'environnement imposées à cette structure. Cette situation inhérente et le prix de la matière première ne permettent plus aux jeunes fabricants et surtout aux producteurs à revenus modestes de faire des économies tant l'argent perçu après commercialisation est insuffisant. Mais le manque d'amélioration de la situation fait que ces jeunes acteurs sont obligés d'abandonner pure et simplement le métier.

Or, ces producteurs, de 2000 à 2014 étaient dans une posture constance de suffisance. La commercialisation du kabakrou était une situation qui faisait d'eux des salariés à part entière. Aussi, ils pouvaient thésauriser, entreprendre et répondre aux besoins des familles. Mais au cours des années 2014 à 2016, il va se produire sous différents motifs, la mévente du savon kabakrou.

D'abord, la Concurrence constante avec le savon liquide traditionnel mis sur le marché. Ce nouveau produit de type traditionnel et parfumé,

\footnotetext{
${ }^{49}$ Données d'enquête 2014
} 
semblable à ceux des supermarchés, a inondé les marchés de Bouaké et attire de plus en plus les ménages qui s’y adonnent à cœur joie. Ensuite, l’odeur qui se dégage du savon kabakrou fait à base de résidus d'huile issu de l'oheol, n'est plus au goût de la clientèle. En effet, les clients trouvent le kabakrou fait à base d'huile de palme meilleur et inodore à contrario de celui fait à base de résidu d'huile issu de l'usine dégageant une forte odeur après usage. Les conséquences sont immédiates :

Premièrement, l’on assiste à l'abandon progressif de la fabrication du kabakrou par certains acteurs, faute de moyens pour l'achat de l'huile de palme qui semble-t-il, être de plus en plus préférée par les ménages, au profit du savon liquide de type traditionnel et parfumé (3L à 500fcfa).

Deuxièmement, il est de plus en plus constaté l'importation hebdomadaire, de l'huile de palme des régions sud et ouest de la Côte d'Ivoire. Cette situation très expansive ne fait que renforcer la condition miséreuse des producteurs de kabakrou, vu que les barils d'huile de palme sont estimés soit 20.000, soit 30.000Fcfa ${ }^{50}$.

Cette situation a des répercussions négatives sur la commercialisation du kabakrou et sur les fabricants de Gbintou. En effet, ils n’ont plus les moyens pour satisfaire leurs moindres besoins et ne peuvent plus faire face à la scolarisation de leurs enfants, à défaut d'écouler leurs productions. A cet effet, ils accusent également un retard dans le paiement de leur commande d'huile de palme. Il faut noter que cette situation d'inconfort les renoue peu à peu à la pauvreté et au découragement. Ainsi, affirme Dième «Tous nos projets se sont amenuisés. Cette condition miséreuse, ne nous arrange pas, mon petit, car nous avons beaucoup de besoins à satisfaire : le paiement des factures d'eau et d'électricité; le salaire des ouvriers à pourvoir ; les besoins nutritionnels et sanitaires de nos familles à satisfaire. » Or, la quasitotalité de ces producteurs de kabakrou sont pères ou mères de plusieurs enfants. Il est sans ignorer qu'en Afrique et en Côte d'Ivoire ; celui qui exerce, travaille pour le lignage; il est tenu de répondre à toutes les sollicitations qui affluent de partout. A cette situation vient se greffer les difficultés sanitaires des acteurs du kabakrou.

\section{Les contraintes sanitaires}

La fabrication du kabakrou est source formelle de revenus. Mais ces acteurs sont de plus en plus confrontés aux contraintes d’ordre sanitaires, ce que confirment les enquêtés : sanitaire $^{51}$.

La fabrication du kabakrou est devenue source de nuisance

\footnotetext{
${ }^{50}$ Données d'enquêtes 2014

${ }^{51}$ Homme de nationalité malienne, 51 ans.
} 
Des maladies telles: les affections broncho-pulmonaires, cutanées, oculaires, l'anémie et

les accidents (brulures) dans le travail sont de plus en plus nombreux et fréquents pendant

la fabrication $^{52}$.

Ces propos révèlent l'existence effective de risques sanitaires dans le travail auxquels s'exposent toujours les fabricants, au cours de la cuisson et de la modélisation du savon. Car la soude caustique en solution est un produit industriel corrosif et très toxique. La simple respiration de ses émanations peut avoir un effet nocif sur les poumons. Tous ceux qui sont d'une manière directe ou indirecte en contact permanente avec la soude doivent connaître et appliquer des procédures de sécurité strictes. Elles sont tenues de se protéger tout le corps, et pratiquer cette activité dans des espaces éloignés de tous lieux d'habitation, soutient d'ESSO ERIC ${ }^{53}$.

La soude qui a l'aspect de l'eau, n'a pas d'odeur, ni de couleur. De ce fait, celui qui l'utilise ne peut être averti du danger qu'elle présente. Un contact avec les yeux suffit pour causer des lésions permanentes, voire la cécité. Avant de ressentir la brûlure, elle a déjà irrité une bonne partie de votre corps ${ }^{54}$.

La soude est reconnue pour sa dangerosité et ses effets nocifs sur la santé. Terre des hommes abonde dans le même sens ${ }^{55}$ :

En guinée, des activités génératrices de revenus qui se développent au sein des ménages impliquent parfois l'utilisation sans précautions particulières de produits toxiques, hautement dangereux. Ainsi, la production artisanale de savon s'est multipliée dans les foyers pauvres et défavorisés, sans s'accompagner de mesures de sécurité indispensables pour éviter l'absorption accidentelle des produits chimiques par les enfants...Chaque année en Guinée, entre 100 et 300 enfants sont victimes d'une ingestion non intentionnelle de soude caustique sans soins adéquats, un tel accident peut causer la mort.

Il ressort des données du terrain, plusieurs causes liées aux problèmes de santé. Tel, l'exposition au feu, durant les étapes de la transformation du savon. Des heures durant, les acteurs sont en contact permanent avec la chaleur. Pour vérifier et mélanger l'huile recueillie avec la soude caustique

\footnotetext{
${ }^{52}$ Femme de nationalité burkinabé, 42 ans. Les brûlures se manifestent par des irritations, et une impression « d'onctuosité » sur la peau comme, si elle était couverte d'une fine pellicule grasse. Toute brûlure même minime qui serait mal traitée peut entrainer de graves lésions.

53 Maitre de conférences en biologie-chimie, Université Felix Houphouët Boigny de cocody.

${ }^{54}$ ALOYSE, Kouao : Enquête express : Fabrication artisanale du savon kabakrou, Abidjan, Le Sursaut, 2014.

55 Terre des hommes: ''Guinée, le fléau de la soude caustique’’, 21 Février, 2014, www.tdh.ch/fr/news/guinee-fleau-soude, consulté le 20 avril 2015, à 18 h15 mn.
} 
pour l'obtention d'une pâte homogène. Outre la chaleur et la fumée, les gaz qui se dégagent de la combustion et de la cuisson, provoquent les affections broncho-pulmonaires, cutanées, oculaires et l'anémie.

Dans certains cas, l'anémie est causée par la longue présence près du feu. Cette pathologie se manifeste généralement par les vertiges, les tremblements des extrémités du corps (les mains, les pieds) avec paresthésie $^{56}$, la sècheresse cutanée et le plus souvent la desquamation de la peau. L'implication de la fumée et des gaz dans les pathologies est perceptible dans les activités professionnelles de façon générale. Tel est le cas de la fabrication du savon kabakrou. En effet, la combustion du bois de chauffe utilisé dans la cuisson du savon de kabakrou produit de la fumée, mélange de produits gazeux et de particules solides de couleurs variables, toxique pour l'organisme. De même, sous l'action du feu, la vapeur d'eau qui se dégage de la transformation du savon kabakrou contient des acides oxalique et cyanhydrique dangereux pour la santé. Constamment inhalés, ces gaz se déposent à une vitesse effrénée dans les voies respiratoires. Ces corps étrangers empêchent le fonctionnement normal des alvéoles. Certains organismes (microphages) interviennent pour détruire ces éléments extérieurs à l'organisme, provoquant la toux, l'asthme etc. GOSSAN justifie leur implication dans les affections oculaires :

Ces gaz (fumée, vapeur d'eau), provoquent également la conjonctivite. Corps étrangers toxiques, en pénétrant dans les yeux, ils irritent le cristallin, les membranes, la cornée, ce qui provoque une réaction électro visuelle, caractérisée par des larmoiements ou écoulements en abondance de larmes. La fumée, la vapeur d'eau, la poussière sont des éléments extérieurs, qui s'introduisent dans les yeux et provoquent des irritations, des larmoiements. ${ }^{57}$

Sous l'action répétée de la chaleur, les ions, contenus dans le sang, particulièrement le magnésium et le calcium, sortent de l'organisme, à travers la sudation (transpiration). Affaiblie par les agressions répétées, la peau n'arrive plus à protéger l'organisme, ce qui constitue une porte ouverte aux maladies dont l'anémie (tremblement des doigts, dessèchement de la peau). Des travaux scientifiques ont justifié la responsabilité de la fumée dans les maladies professionnelles, à l'instar du travail de kabakrou.

MISHRA soutient que plusieurs foyers des pays en développement font usage de biocombustibles (bois, fumier, paille) pour cuisiner et se chauffer. Ainsi, environ 3,5 milliards de personnes habitant surtout en zone rurale se trouvent exposées chez elles à des niveaux élevés de polluants

\footnotetext{
${ }^{56}$ La paresthésie est le nom scientifique de la sensation de fourmillements, engourdissements ou autres picotements pouvant être ressentis dans diverses parties du corps, en particulier les membres et leurs extrémités (bras, mains, jambes, pieds).

${ }^{57}$ Homme 38 ans, infirmier.
} 
aériens. L'exposition prolongée à des composants toxiques est néfaste pour le système respiratoire, les yeux et le système immunitaire. Ils fragilise l'organisme face aux infections et aux maladies. Cette pollution peut entrainer de graves problèmes de santé (tuberculose, infections respiratoires aigües). Associée à l'asthme, elle peut provoquer l'anémie et la cécité ${ }^{58}$.

Face à la maladie, les producteurs utilisent différents voies thérapeutiques pour recouvrer la santé. Lorsque leur état de bien-être physique et mental est menacé, le premier réflexe des producteurs consiste à recourir à l'automédication. A ces traitements, s'ajoutent les plantes médicinales ${ }^{59}$, dont la décoction. Face aux échecs, et à la détérioration de leur état de santé, ils s'orientent vers les centres de santé ${ }^{60}$. Mais, l'existence d'accidents de travail (brûlures) et de maladies (anémie, affections bronchopulmonaires) doivent interpeller les autorités et les producteurs sur les conditions de travail et les dangers liés à la fabrication du savon traditionnel dans les quartiers précaires comme Gbintou.

\section{Conclusion}

Au terme de cette étude, nous pouvons retenir que si la fabrication du savon traditionnel kabakrou est demeurée aujourd'hui dans l'impasse à Gbintou (rencontre des difficultés), c'est parce que plusieurs facteurs le justifient :

- d'abord, les difficultés d'approvisionnement en matières premières motivées par les questions environnementales et économiques ;

- ensuite, la mévente progressive du savon traditionnel kabakrou due au savon liquide traditionnel qui a envahi le marché de Bouaké ;

- enfin, les contraintes sanitaires qu'entrainent de plus en plus la fabrication du savon kabakrou.

Bref, la fabrication du savon traditionnel kabakrou à Gbintou a certes joué des rôles économiques d'intérêts personnels. Mais, avec les difficultés de leurs jours, les acteurs gagneraient à s'organiser en coopérative pour mieux contrôler tout le processus de fabrication et de vente. De la sorte, une politique d'approvisionnement est souhaitée pour satisfaire les parties prenantes en les mettant toutes à contribution, de sorte que si un problème éclate, elles puissent trouver la mesure et la solution.

\footnotetext{
${ }^{58}$ MISHRA, Vinod : Fumée et feux, www.ourplanet.com/imgvern/122/french/mishra.html, consulté le 22 Avril 2015, à 16h.

${ }^{59}$ Feuilles de papayer, de goyavier...

${ }^{60}$ Infirmerie privée située à Djézoukouamé, près du Lycée la Renaissance, à l’arrière de l’Eglise UEESO-CI.
} 


\section{References:}

1. AKMEL, Meless Siméon: «Exploitation du charbon de bois et risques sanitaires en pays odjukru ", in European Scientifique Journal, n³0, vol. 8, Macédoine, Décembre, 2012.

2. AKOUETTE, Adrien: Assurer des conditions de travail décentes dans l'économie informelle en Afrique, Johannesburg, CSI-Afrique, 2010.

3. CHANDA, Tithankan: Le chômage des jeunes en Afrique, une génération perdue ? RFI, 24 janvier, 2014.

4. ALOYSE, Kouao: Enquête express: Fabrication artisanale du savon kabakrou, Abidjan, Le Sursaut, 2014.

5. BIRAGO, Diop, Les contes d'Amadou Koumba, Dakar, Présence Africaine, 1961.

6. CROZIER, Michel et FRIEDBERG, Erhard: L'acteur et le système, Paris, Seuil, 1977.

7. CUONZO, Teresa Maria : Les petits métiers: le secteur de l'économie informelle en Afrique, Italie, Université Degli Studi Mediterranea, 2003.

8. DURKHEIM Emile : Les règles de la méthode sociologique, Paris, PUF, 1987.

9. MALIKWISHA, Meni : "L'importance du secteur informel en RDC", in Bulletin de l'ANSD, Volume 1, Congo, Université de Kinshasa, 2000.

10. MARX, Karl : Contribution à la critique de l'économie politique, Paris, Editions Sociales, 1972.

11. MASLOW, Abraham: La théorie de la motivation humaine in Psychological Review, Paris, Eyrolles, 1943.

12. MERTON, Robert King: Eléménts de théorie et de méthode sociologique, Paris, Plon, 1965.

13. MESSING, Karen: La santé des travailleurs. La science est-elle aveugle ? Montréal, Remue-ménage, 2000.

14. MISHRA, Vinod: Fumée et feux, www.ourplanet.com /imgvern/122/french/mishra.html, consulté le 22 Avril 2015, à 16h.

15. MOUSTAPHA, Diabaté: Indicamétrie Capacitaire, Bouaké, CUMERFI, 1999.

16. OLIVIER de Sardan: Anthropologie et développement, Essai en Anthropologie de changement social Kartala, Paris, 1995a

17. QUIVY, Raymond; CAMPEHOUDT, Luc Van: Manuel de recherche en sciences sociales, Paris, Dunod, 2006.

18. SHOMBA, Lomani : La Promotion de l'économie informelle en droit Congolais: quelle option lever entre sa reforme et sa formalisation? Mémoire de Licence en Droit, Congo, Université de Kinshasa, 2003. 
19. SOROKIN, Pitirim : Social and cultural mobility, New York, Free Press, 1959.

20. VOGEL, Laurent: La santé des femmes au travail en Europe, des inégalités non reconnues, Bruxelles, Bureau Technique Syndical Européen sur la santé et la sécurité, 2003.

21. VOM KOLKE, Georg ; KERN Matthias, «Des produits chimiques pour les plus pauvres, risque ou chance ?», in Développement Economique en milieu rural, vol. 13, n¹, Wageningen, CTA, 2006. 